\title{
LAS EMOCIONES Y LA ENSEÑANZA VIRTUAL DE PERSONAS ADULTAS
}

La condición adulta es una realidad personal llena de potencialidades que necesitan desplegarse para propiciar el crecimiento interior y el enriquecimiento de los vínculos sociales. La e-learning se presenta como un medio de incalculable repercusión en el acrecentamiento del valor de las conductas emocionalmente positivas siempre y cuando los comportamientos emocionalmente negativos sean detectados y tratados inicialmente en contacto directo y personal con el alumno.

Palabras clave: Formación de adultos, condición adulta, e-learning, emociones

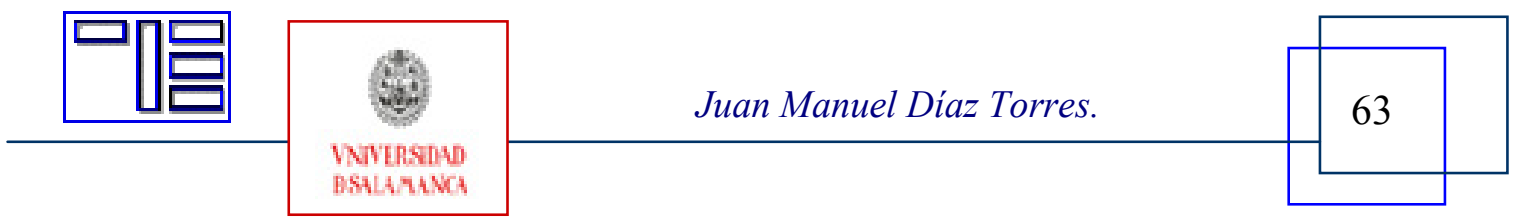


Revista Electrónica Teoría de la Educación.

Educación y Cultura en la Sociedad de la Información.

http://www.usal.es/teoriaeducacion

Vol. 7. No2. Diciembre 2006

\section{THE EMOTIONS AND THE VIRTUAL EDUCATION OF ADULT PEOPLE}

The adult condition is a full personal reality of potentialities that need to unfold to cause the inner growth and the enrichment of the social bonds. E-learning appears like means of incalculable repercussion in the increase of the value of the emotionally positive conducts as long as the emotionally negative behaviors are detected and dealt initially in direct bonding and personal with the student.

Key words: Formation of adults, adult condition, e-learning, emotions

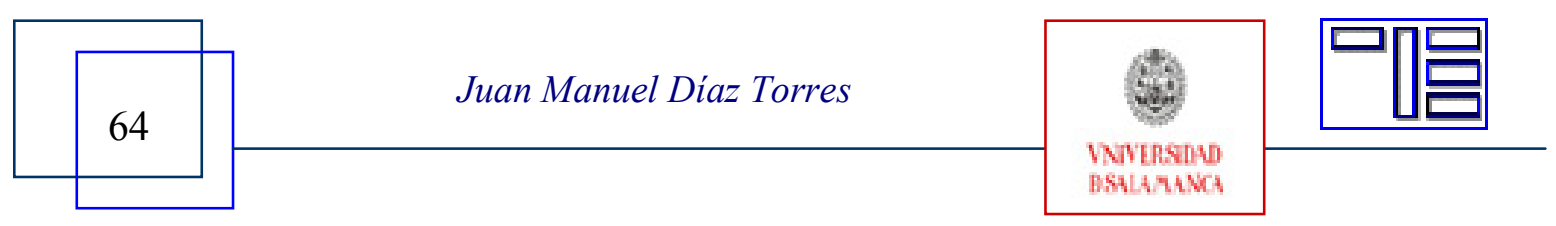




\section{LES ÉMOTIONS ET L'ENSEIGNEMENT VIRTUEL DE PERSONNES ADUL-} TES

La condition adulte est une réalité personnelle inondation de potentialités qui ont besoin d'être dévoilées pour rendre propice la croissance intérieure et l'enrichissement des liens sociaux. L'e-learning se présente comme un moyen de répercussion incalculable dans l'acrecentamiento de la valeur des conduites émotionnellement positives pourvu que les comportements émotionnellement négatifs soient détectés et traités initialement en contact direct et personnel avec l'élève.

Mots clés: Mots clef : Formation d'adultes, condition adulte, e-learning, émotions.

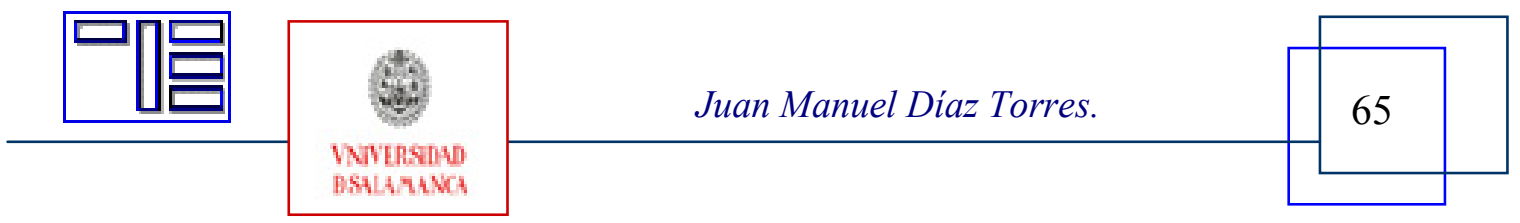


Revista Electrónica Teoría de la Educación.

Educación y Cultura en la Sociedad de la Información.

http://www.usal.es/teoriaeducacion

Vol. 7. No2. Diciembre 2006

\section{LAS EMOCIONES Y LA ENSEÑANZA VIRTUAL DE PERSONAS ADULTAS}

Prof. Juan Manuel Díaz Torres

jmdiazt@ull.es

Universidad de La Laguna.

\section{1.- ENSEÑANZA VIRTUAL DE ADULTOS}

La enseñanza virtual, llamada también e-learning, es la forma de aprender y de enseñar a través de las nuevas tecnologías de la información y la comunicación, especialmente Internet. Dicho proceso se lleva a cabo por medio de herramientas de comunicación y de presentación de contenidos. Entre las primeras destacan el correo electrónico y los foros, mientras que las segundas se muestran a través de textos, gráficos, animaciones y vídeos.

Dichos medios tecnológicos permiten la autorregulación del ritmo de aprendizaje por parte del propio alumno así como la plena disponibilidad para utilizar las herramientas de aprendizaje independientemente de límites temporales o espaciales.

Debe entenderse que la enseñanza virtual abarca tanto el aprendizaje sincrónico como el asincrónico, por lo que aunque en la actualidad se insista más en la enseñanza virtual tutorizada, mixta, combinada o b-learning -blended learning-, que es simultáneamente presencial y virtual, suele estimarse que ésta no representa más que una distinción metodológica dentro de la e-learning.

En general, tanto en el ámbito de la comunicación sincrónica -que es aquella que se establece entre dos o más personas cuando existe coincidencia temporal e interacción directa entre los participantes, ya sea de forma presencial o con separación física-, como en el de la comunicación asincrónica -que es la comunicación que se establece entre dos o más personas de manera diferida, esto es, cuando no existe coincidencia temporal y que permite a cada participante estudiar de forma independiente- se brindan variadas e interesantes perspectivas en el ámbito del conjunto de procesos de desarrollo de técnicas

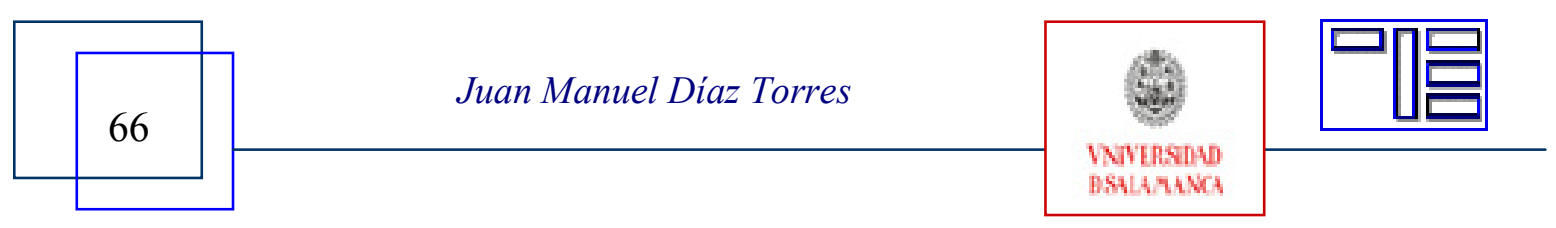


y metodologías que facilitan la formación de personas mayores de edad o que han superado la edad máxima de escolarización obligatoria.

Mediante la enseñanza de personas adultas se pretende incorporar a este colectivo, con sus condicionantes específicos, a un programa de enseñanza institucionalizada a fin de enriquecer sus capacidades y habilidades, ampliar sus conocimientos y mejorar sus competencias técnico-profesionales, de manera que se vean favorecidas por lo que se refiere no sólo a su desarrollo personal y bienestar, sino también a sus niveles de participación cívica y su acceso a la educación y a los bienes culturales en general.

Pues bien, la enseñanza virtual se presenta hoy como la más adaptable a la situación concreta del adulto, una persona que cuando es estudiante no suele serlo en exclusiva ni a tiempo completo.

Aunque el perfil del adulto que retorna o se inicia en los estudios oscila entre la de aquellos que no tuvieron acceso a los estudios y la de quienes, habiéndolo tenido, no hicieron uso de tal derecho, la sistematización de los motivos que llevan a un adulto a volver a dedicar tiempo a unos estudios o a iniciarlos no es tarea sencilla si lo que se pretende es trascender una mera casuística más o menos clasificada.

En general las perspectivas culturales del adulto que se siente impelido a aprender no son siempre limitadas o delineadas por una determinada actividad profesional u ocupación. Tal persona puede tener necesidades formativas o culturales concretas no coincidentes con las que se circunscriben a su actividad profesional u ocupación. De hecho, resulta incesante la generación de nuevos contextos y necesidades que de alguna manera constriñen al adulto a desenvolverse con gran dinamismo.

Lo cierto es que el instante en el que una persona adulta toma la decisión de dedicar parte de su tiempo al estudio es el momento cumbre de una necesidad interior largamente insatisfecha, y que tal necesidad formativa suele venir dada, generalmente, por el afán de mejorar.

El adulto que es objeto de nuestra atención es aquel que necesita o desea acceder a estudios superiores y que lleva tiempo desvinculado del rol de estudiante, y cuya nueva ocupación -que le hará ponerse a prueba en cuanto a su capacidad intelectual, fuerza de voluntad y habilidades procedimentales- le forzará a reorganizar tanto su tiempo libre como su tiempo familiar y profesional.

La importancia de la enseñanza virtual de adultos está sobradamente reconocida y sus bases establecidas en sus líneas más generales. Lo que sigue siendo pertinente en ahondar en la condición imprescindible para que todo lo demás tenga sentido, esto es, en el adulto motivado en función del cual se elaboran ofertas educativas y programas de estudio.

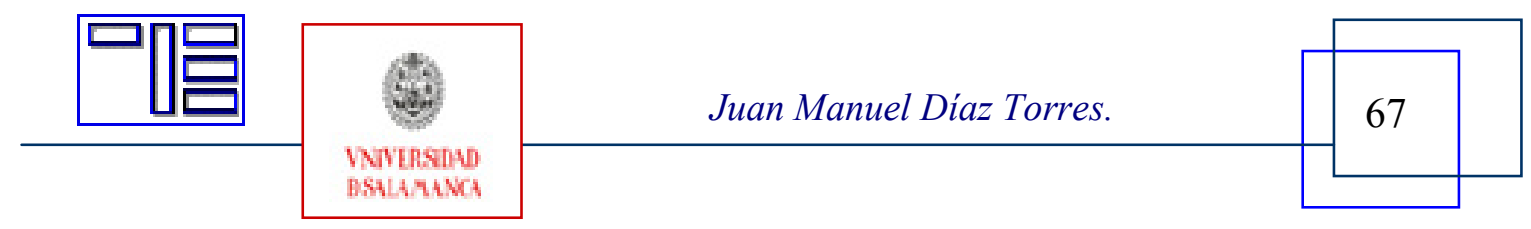


Revista Electrónica Teoría de la Educación.

Educación y Cultura en la Sociedad de la Información.

http://www.usal.es/teoriaeducacion

Vol. 7. No2. Diciembre 2006

\section{2.- LAS NUEVAS TECNOLOGÍAS EN EL AULA}

Dadas las dificultades más significativas que confluyen en la persona adulta a la hora de someterse a un proceso estructurado de aprendizaje sostenido en el tiempo, cabe afirmar que el adulto parece hallar su lugar apropiado en una institución de enseñanza que le permita autorregularse espacio-temporalmente no sólo en cuanto a los lugares y a los momentos que va a dedicar a su preparación sino también por lo que se refiere al ritmo de estudio.

Los procedimientos y los procesos en la enseñanza virtual permiten al adulto la recepción de apoyo, la facilitación del aprendizaje y la orientación por parte del equipo docente, además de posibilitar la reducción del impacto negativo que la actividad formativa tiene sobre sus hábitos profesionales, familiares e individuales y, además, alentar un diálogo interior entre las experiencias y conocimientos previos de quien ya está suficientemente preparado para la vida y la realidad emergente.

En general, se ha afirmado (Marchesi y Martín, 2004) que las ventajas de la utilización de las nuevas tecnologías en el aula -ventajas potenciales, pues sólo pueden desplegarse como auténticas posibilidades realizables cuando el modelo educativo en el que se inscribe su utilización propicia un ambiente de construcción del conocimiento- son, básicamente, las siguientes:

1.Incentivan la creación de contextos de aprendizaje que suponen la inauguración de nuevas posibilidades de información y de comunicación;

2.Facilitan la aproximación de la escuela a entornos educativamente no formales como son la familia y los amigos, incrementándose la transferencia de los aprendizajes obtenidos en contextos formales;

3.Simplifican la comprensión y el aprendizaje de nociones abstractas al permitir representarlas figurativamente;

4.Posibilitan la interactividad al favorecer que los estudiantes se adentren en experiencias de aprendizaje en las que no sólo se limitan a recibir nueva información sino que, además, entren en contacto con otros discentes y docentes, pudiendo comprobar sus propios avances y dificultades a la vez que ensayar diversas estrategias para ir construyendo sus conocimientos.

Si bien los espacios virtuales en general y la enseñanza virtual en concreto pueden resultar medios excelentes de enseñanza-aprendizaje, recaen ciertas dudas acerca de que puedan serlo con independencia de tres factores: en primer lugar, de la difuminación del magisterio personal, espacial, directo, cara a cara; en segundo lugar, de la mediación virtual en la compensación de las deficiencias individuales, sociales y económicas; y, en tercer lugar, de la eticidad que ha de acompañar a las estructuras cognitivas.

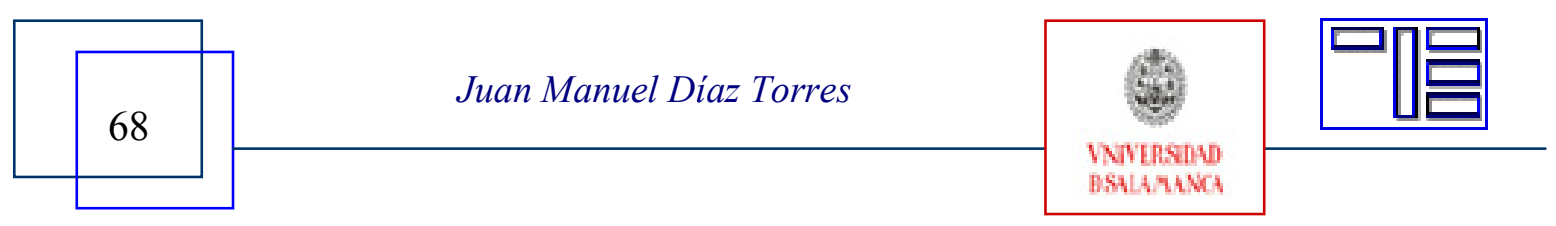




\section{3.- FACTORES CONDICIONANTES DEL APRENDIZAJE DEL ADULTO}

El proceso de aprendizaje en la persona adulta se mueve dentro de unas coordenadas determinadas, definidas precisamente por la condición de ser adulto. De tal condición derivan también las dificultades u obstáculos.

El aprendizaje del adulto parece guiarse por condicionantes intrínsecos -como la necesidad sentida o el anhelo insatisfecho- más que por los de naturaleza extrínseca -tales como la presión ambiental o las exigencias sociales-, yendo por ello mismo más allá de la mera integración de conocimientos. En el fondo, el aprendizaje en personas adultas pretende más bien la integración de niveles con el fin de optimizar la personalidad.

No son pocos los autores que han intentado elaborar un catálogo de los factores condicionantes del aprendizaje del adulto (Palladino, 1981; Wedemeyer, 1981; García Aretio, 1996). Entre los factores que obstaculizan la labor de aprendizaje y exigen, por tanto, un esfuerzo suplementario se encuentran los siguientes:

1.La heterogeneidad en edad, intereses, ocupaciones, motivaciones, experiencias y aspiraciones del potencial colectivo de adultos en sesiones grupales;

2.La marginalidad de la actividad de aprendizaje, que resulta siempre una ocupación no sólo inestable sino, también, secundaria o posterior con respecto a otras actividades y compromisos;

3.Cansancio y la escasez de tiempo, esto es, una disminución de las energías vitales derivadas de lo anterior;

4.La claridad y voluntariedad de los objetivos propuestos; no debe olvidarse que la persona adulta basa toda su actividad de aprendizaje no en la obligación sino en el convencimiento.

5.Disminución de la ductilidad adaptativa ante el cambio de perspectivas teóricas y prácticas, derivada del bagaje experiencial -emocional y comportamental- y cognoscitivo - falta de hábitos de estudio y olvido de entramados conceptuales y teóricos-;

6.Lentitud en el aprendizaje por su tendencia a querer entender, relacionar y aplicar lo estudiado.

7.El deseo intenso de resultados positivos;

8.Un elevado grado de ansiedad ante la posibilidad de la pérdida de tiempo y el fracaso;

9.Una alta susceptibilidad y desprotección ante las observaciones y las críticas;

10. Necesidad de verse reforzado en el concepto de sí, pues la autoestima del adulto que estudia es muy frágil. La edad, las experiencias negativas pasadas, una excesiva autoexigencia además de deficiencias en técnicas de estudio y posibles limitaciones cognoscitivas marcan indeleblemente este período vital y académico.

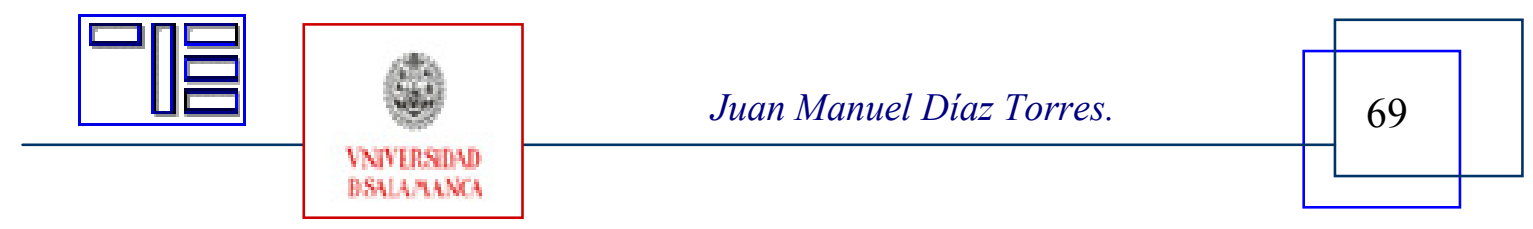


Revista Electrónica Teoría de la Educación.

Educación y Cultura en la Sociedad de la Información.

http://www.usal.es/teoriaeducacion

Vol. 7. No2. Diciembre 2006

\section{4.- CONCLUSIONES}

Como puede observarse, son los cuatro últimos factores los que pertenecen al ámbito de lo emocional: el deseo intenso de resultados positivos, el elevado grado de ansiedad ante la posibilidad de la pérdida de tiempo y el fracaso, una alta susceptibilidad y desprotección ante las observaciones y las críticas y una situación crítica de la autoestima de fragilización del concepto de sí.

Si se consideran más atentamente se llega a la conclusión de la centralidad del último de ellos, con respecto al cual los demás resultan derivados $\mathrm{y}$, por ello, periféricos respecto de aquél. Desmotivación, fracaso y desprotección conforman, pues, el entramado potencialmente pernicioso respecto de la autoestima.

De ello puede deducirse que en la enseñanza superior virtual de adultos no puede quedar desatendido el aspecto del fortalecimiento de la autoestima. Sin embargo, ni siquiera la cuarta ventaja de la utilización de las nuevas tecnologías en el aula, citada más arriba, deja de soslayar este aspecto. La posibilidad de la interactuación o comunicación virtual intersubjetiva resulta excesivamente cognitivista, a pesar de incidir directamente en aspectos emocionales como son la generación de ritmos interiores comunes y el acompasamiento de nuevas experiencias.

Todo ello invita a un replanteamiento de la e-learning con adultos en educación superior dirigido a enfatizar la necesidad no sólo de la atención tutorial real o cara a cara sino, también, la de generar procesos de mejora sensible a través de una evaluación continua que permita al adulto discente ir comprobando la adecuación entre su trabajo intelectual y los objetivos de cada una de los temas o unidades didácticas.

Por lo que se refiere a la atención tutorial real o cara a cara hay que decir que es en ella donde, en gran medida, se fortalece la autoestima del alumno adulto al facilitar la detección de crisis emocionales. Y en relación a la evaluación continua, hay que decir que esta permite observar no sólo el grado de adquisición de los conocimientos sino algo que es mucho más importante como es la tendencia cognitiva y emocional del estudiante.

La condición adulta se presenta, pues, como una realidad personal cargada de potencialidades que necesitan desplegarse a fin de propiciar un mayor crecimiento interior y un enriquecimiento de los vínculos convivenciales.

La e-learning se presenta, así, como un medio de incalculable repercusión en el acrecentamiento del valor de las conductas emocionalmente positivas siempre y cuando los comportamientos emocionalmente negativos o críticos sean detectados y tratados inicialmente en contacto directo y personal con el alumno.

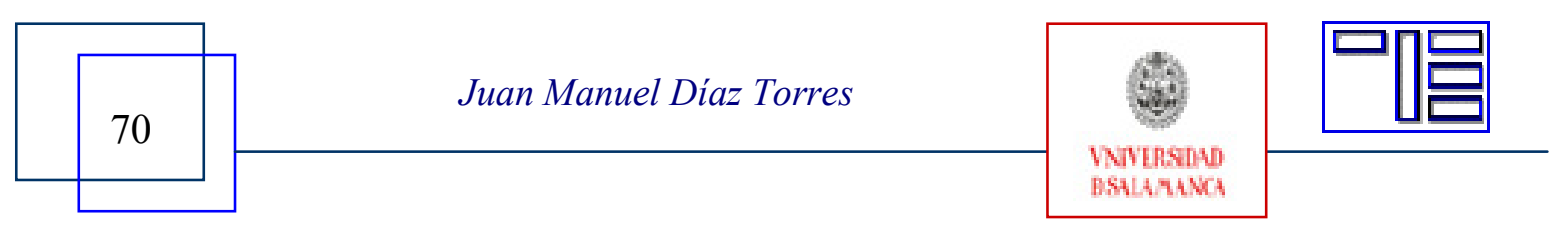




\section{5.- BIBLIOGRAFÍA}

ADELL, J. (1993): World Wide Web: Un sistema hipermedia distribuido para la docencia universitaria, en BLÁZQUEZ, F., CABERO, J. y LOSCERTALES, F. (Coord.) (1994): Nuevas tecnologías de la Información y la Comunicación para la Educación. Sevilla, Ediciones Alfar. http://nti.uji.es/docs/nti/badajoz.html

ADELL, J. (2003): Comunidades de aprendizaje en la formación presencial: más allá del curso online. Actas de las I Jornadas Canarias sobre las TIC en la Docencia Universitaria. La Laguna - Las Palmas de Gran Canaria.

http://www.edulab.ull.es/jornadas/actas.asp

ALONSO, C. y GALLEGO, D. (2002): Tecnologías de la Información y la Comunicación. http://dewey.uab.es/pmarques/EVTE/algatic.doc

AREA, M. (1998): Desigualdades, educación y nuevas tecnologías. http://tecnologiaedu.us.es/bibliovir/pdf/a5.pdf

AREA, M. (2001): La oferta de educación superior a través de Internet. Análisis de los Campus Virtuales de las universidades españolas. Madrid, Dirección General de Universidades - Ministerio de Educación, Cultura y Deporte.

AREA, M. (2002): Sociedad de la información y analfabetismo tecnológico: nuevos retos para la educación de adultos. http://tecnologiaedu.us.es/bibliovir/pdf/a10.pdf ; también en http://webpages.ull.es/users/manarea/Documentos/documento10.htm

BARTOLOMÉ, A. (1999): Nuevas tecnologías en el aula. Guía de supervivencia. Barcelona, GRAÓ-ICE Universidad de Barcelona.

BRAVO RAMOS, J. L.; SÁNCHEZ NÚÑEZ, J. A. Y FARJAS ABADÍA, M.: El uso de sistemas de b-learning en la enseñanza universitaria.

http://dewey.uab.es/pmarques/pdigital/simo/juanlbravo.doc

CABERO, J. y otros (1999): Nuevas Tecnologías en la formación flexible y a distancia. Sevilla, Kronos.

CABERO, J. y otros (2000): Nuevas Tecnologías aplicadas a la Educación. Madrid, Síntesis.

CIRIGLIANO, G. F. J. (1983): La educación abierta. Buenos Aires, El Ateneo.

DOOLITTLE, P. E. (1999): Constructivism and Online Education

http://edpsychserver.es.vt.edu/workshops/tohe1999/online.html

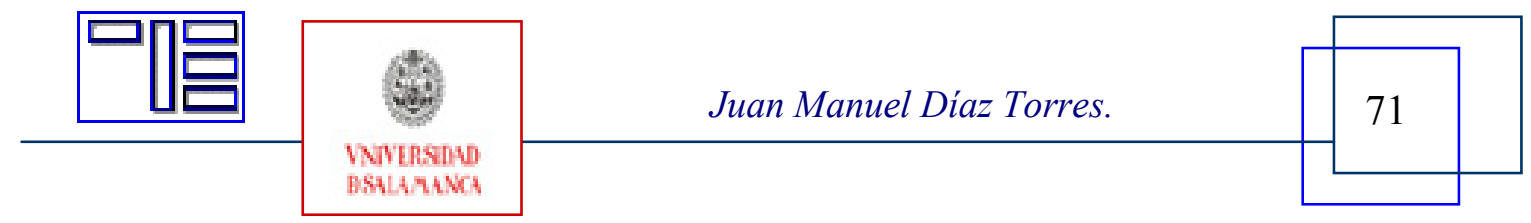


Revista Electrónica Teoría de la Educación.

Educación y Cultura en la Sociedad de la Información.

http://www.usal.es/teoriaeducacion

Vol. 7. No2. Diciembre 2006

FAINHOLC, B. (2003): Los nuevos retos de la educación y la formación en la sociedad de la información. http://dewey.uab.es/pmarques/EVTE/fainnuevosretos.doc

FARJAS, A.; MADRIGAL, C.; NOGALES, E. (1996a): La UNED y sus alumnos, en GARCÍA ARETIO, L. (Coord.): La educación a distancia y la UNED. Madrid, Universidad Nacional de Educación a Distancia, 413-471

GARCÍA ARETIO, L. (1987): Rendimiento académico y abandono en la educación superior a distancia. Madrid, ICE-UNED.

GARCÍA ARETIO, L. (1994): Educación a distancia hoy. Madrid, Universidad Nacional de Educación a Distancia.

GARCÍA ARETIO, L. (1996): El aprendizaje a distancia de las personas adultas, en GARCÍA ARETIO, L. (Coord.): La educación a distancia y la UNED. Madrid, Universidad Nacional de Educación a Distancia, 81-126

GARCÍA ARETIO, L. (Coord.) (1997): Investigar para mejorar la calidad de la Universidad. Trabajos de investigación sobre la UNED. Madrid, Universidad Nacional de Educación a Distancia.

GONZÁLEZ SOTO, A. P. (1996): Hacia un nuevo modelo de formación de personas adultas, en MEDINA, A. y GENTO, S. (Coords.) (1996): Formación de educadores de personas adultas III (Educación Secundaria). Madrid, Universidad Nacional de Educación a Distancia, 145-205.

GROS, B. (2000): El ordenador invisible. Hacia la apropiación del ordenador en la enseñanza. Barcelona, Gedisa.

HARASIM, L. y otros (2000). Redes de aprendizaje. Guía para la enseñanza y el aprendizaje en red. Barcelona, Gedisa.

HERRÁN, A. de la (2003): De la sociedad del acceso a la información a una sociedad mundial y evolutiva. Indagaciones para una reforma profunda de la educación. http://dewey.uab.es/pmarques/EVTE/siherran.pdf

MARCHESI, A. y MARTÍN, E. (Coord.) (2004): Tecnología y aprendizaje. Investigación sobre el impacto del ordenador en el aula. Madrid, Ediciones SM. http://www.piloto.librosvivos.net

MEDINA RIVILLA, A. (Coord.) (1997): Diseño y desarrollo curricular para la formación de las personas adultas. Universidad Nacional de Educación a Distancia. Madrid.

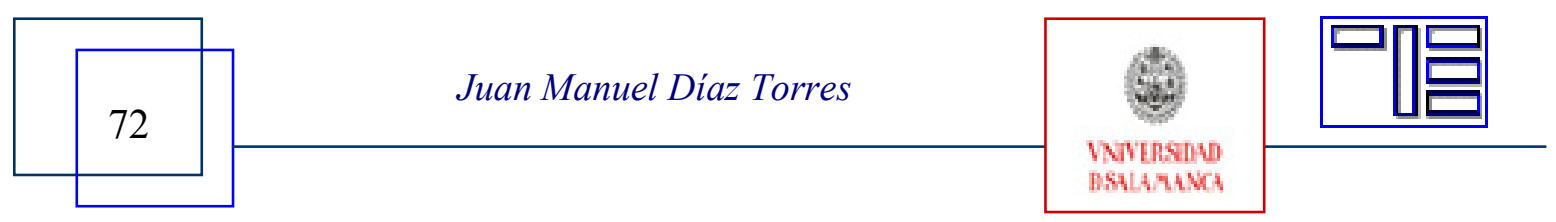


ORTEGA, J. A. (2001): Nuevas tecnologías y compensación de desigualdades educativas: Hacia un compromiso de solidaridad en la formación de los inalcanzados y los excluidos. Melilla, Actas del Congreso Nacional sobre Aspectos Didácticos y Organizativos de la Educación. http://www.ugr.es/ sevimeco/biblioteca/tecnologias/Ntcde.htm

PALLADINO, E. (1981): Educación de adultos. Buenos Aires, Humanitas.

UNESCO (1998): Aprendizaje abierto y a distancia. Perspectivas y consideraciones políticas. Madrid. Universidad Nacional de Educación a Distancia.

VÍLCHEZ, L. F.: Las T.I.C. en el trasfondo emocional y ético de la educación y la interculturalidad. http://www.uv.es/soespe/vilchez.htm

WEDEMEYER, C. A. (1981): Learning at the Back Door. Reflections on NonTraditional Learning in the Lifespan. Madison: University of Wisconsin Press.

\section{Para citar esta artículo puede utilizar la siguiente referencia:}

DÍAZ TORRES, Juan Manuel (2006): Las emociones y la enseñanza virtual de personal adultas. GARCÍA CARRASCO, Joaquín (Coord.) Estudio de los comportamientos emocionales en la red [monográfico en línea]. Revista electrónica Teoría de la Educación: Educación y Cultura en la sociedad de la información. Vol. 7, $\mathrm{n}^{\circ} 2$. Universidad de Salamanca. [Fecha de consulta: dd/mm/aaaa].

$<$ http://www.usal.es/ teoriaeducacion/rev_numero_07_02/n7_02_juan_manuel_diaz_torres.pdf $>$ ISSN 1138-9737

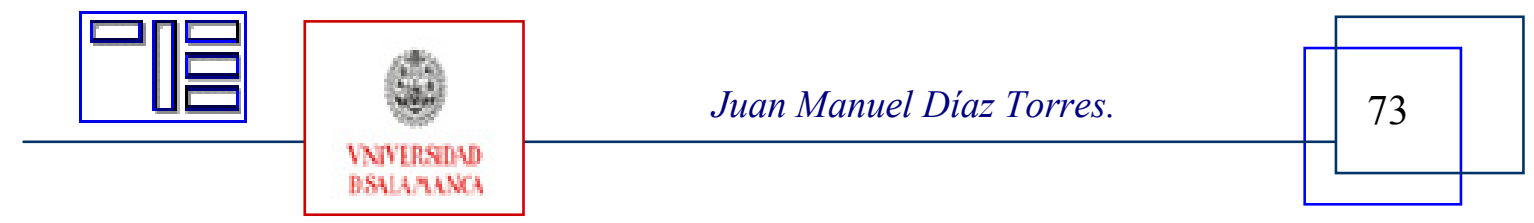

